THE PROBLEM OF THE IONIZED HYDROGEN MOLECULE

By C. Y. ChaO

Norkan Bridge Laboratory of Physics, California Institute of Thchnology

Communicated May 20, 1929

The problem of the ionized hydrogen molecule was first treated by Pauli ${ }^{1}$ who used the old quantum theory and obtained a result which was not in agreement with experiment. Niessen, ${ }^{2}$ however, obtained the experimental value for the lowest energy level by introducing half-quantum numbers. The problem has now been treated by methods of wavemechanics; thus Burrau ${ }^{3}$ obtained a result in very good agreement with experiment by a numerical integration and a general discussion has been given recently by $\mathrm{A}$. $\mathrm{H}$. Wilson. ${ }^{4}$ It is well known that the polynomial method of wave-mechanics, invented by Brillouin and Wentzel and developed by Sommerfeld, ${ }^{5}$ gives, for a certain group of problems, the same result as the old quantum theory in approximation (0). and gives halfquantum numbers in approximation (1). It is shown in this paper that the present problem belongs to this group.

Neglecting the motion of the two nuclei, the wave equation of $\mathrm{H}_{2}^{+}$is

$$
\frac{\partial^{2} \psi}{\partial x^{2}}+\frac{\partial^{2} \psi}{\partial y^{2}}+\frac{\partial^{2} \psi}{\partial z^{2}}+\frac{8 \pi^{2} m_{e}}{h^{2}}\left(E+\frac{e^{2}}{r_{A}}+\frac{e^{2}}{r_{B}}\right) \psi=0,
$$

where $E$ is equal to the total energy minus the energy due to the repulsion of the nuclei (Fig. 1).

Introduce the new coördinates:

and set

$$
\xi=\frac{r_{A}+r_{B}}{r_{A B}}, \eta=\frac{r_{A}-r_{B}}{r_{A B}}, \phi,
$$

$$
\psi=P(\xi) Q(\eta) \Phi(\phi),
$$

we get the following equations:

$$
\begin{gathered}
\frac{d^{2} \Phi}{d \phi^{2}}=-m^{2} \Phi \\
\frac{d}{d \xi}\left\{\left(\xi^{2}-1\right) \frac{d P}{d \xi}\right\}+\left\{-\lambda \xi^{2}+2 \rho \xi+\frac{m^{2}}{\xi^{2}-1}+\mu\right\} P=0, \\
\frac{d}{d \eta}\left\{\left(1-\eta^{2}\right) \frac{d Q}{d \eta}\right\}+\left\{\lambda \eta^{2}+\frac{m^{2}}{1-\eta^{2}}-\mu\right\} Q=0,
\end{gathered}
$$

where $\lambda, \mu, \rho$ are three constants to be determined, which have the following meanings: 


$$
\lambda=-\frac{2 \pi^{2} m_{e} \mathrm{r}^{2} A B E}{h^{2}}=\rho^{2} \frac{E}{4 E_{H}}, \rho=\frac{r_{A B}}{r_{H}} .
$$

Since equation (4) is the well-known spheroidal wave equation whose solutions have been worked out in very simple forms, ${ }^{6}$ we shall use the result of Wilson for the lowest state, i.e.:

$$
\mu=\frac{1}{3} \lambda+\frac{2}{135} \lambda^{2}+\frac{4}{3^{5} \cdot 5 \cdot 7} \lambda^{3}+\frac{16}{3^{7} \cdot 5^{3} \cdot 7^{2}} \lambda^{4}+\ldots
$$

and shall consider here only equation (3). (Besides, the present method does not give very good results for (4) due to the fact that $\left(1-\eta^{2}\right)$ occurs
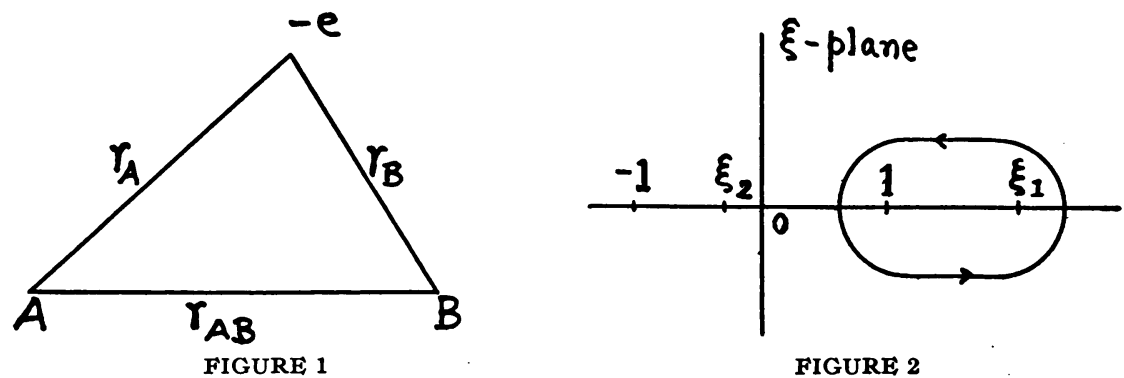

FIGURE 2

in the denominators of the successive approximations.) We shall also set $m=o$; and introduce constants $a, b, c$, which are defined by

$$
\lambda=\frac{4 \pi^{2}}{h^{2}} a, \mu=\frac{4 \pi^{2}}{h^{2}} b, \rho=\frac{4 \pi^{2}}{h^{2}} c .
$$

Now, the equation in $\xi$ for $m=o$ reduces to the following,

$$
\frac{d^{2} P}{d \xi^{2}}+\frac{2 \xi}{\xi^{2}-1} \frac{d P}{d \xi}+\frac{4 \pi^{2}}{h^{2}} \frac{1}{\xi^{2}-1}\left(-a \xi^{2}+2 c \xi+b\right) P=0 .
$$

Let

$$
P=e^{\frac{2 \pi i}{h} \int y d \xi}
$$

we have

$$
\frac{h}{2 \pi i}\left(y^{\prime}+\frac{2 \xi}{\xi^{2}-1} y\right)=-\frac{a f(\xi)}{\xi^{2}-1}-y^{2}
$$

where

$$
f(\xi)=\xi^{2}-\frac{2 c}{a} \xi-\frac{b}{a}=\xi^{2}-\frac{2 \rho}{\lambda} \xi-\frac{\mu}{\lambda} .
$$

Next, we introduce the quantum condition by setting

$$
y=\sum_{\nu=0}^{\infty}\left(\frac{h}{2 \pi i}\right)^{\nu} y^{\nu}, \mathscr{S} y d \xi=n_{1} h .
$$


Substituting the above expression of $y$ into (9), we have

$$
\begin{aligned}
\frac{h}{2 \pi i}\left[y_{0}^{\prime}+\frac{h}{2 \pi i} y_{1}^{\prime}+\ldots\right. & \left.+\frac{2 \xi}{\xi^{2}-1}\left(y_{0}+\frac{h}{2 \pi i} y_{1}+\ldots\right)\right] \\
& =-\frac{a f(\xi)}{\xi^{2}-1}-\left(y_{0}+\frac{h}{2 \pi i} y_{1}+\ldots\right)^{2} .
\end{aligned}
$$

Taking only the terms independent of $h$, we get for the approximation (0),

$$
y_{0}=\sqrt{\frac{-a f(\xi)}{\xi^{2}-1}}, \oint \sqrt{\frac{-a f(\xi)}{\xi^{2}-1}} d \xi=n_{1} h,
$$

which is precisely the result of the old quantum theory.

Using the above expression for $y_{0}$, and equating the first powers of $h$ in (11), we get, for approximation (1),

$$
y_{1}=-\frac{1}{2 y_{0}}\left\{y_{0}^{\prime}+\frac{2 \xi}{\xi^{2}-1} y_{0}\right\}=-\frac{1}{2}\left\{\frac{\xi}{\xi^{2}-1}+\frac{a \xi-c}{a f(\xi)}\right\} .
$$

To find a suitable contour of integration, we first notice that $a$ and $c$ are both positive; we have also from (6), that $b>0$. This corresponds, in the old quantum theory, to the case $\sqrt{\frac{b}{a}} \leqslant \eta \leqslant 1$, which gives a possible equilibrium state for $m=0$ as mentioned in Pauli's paper. Hence we have two roots for the equation $a f(\xi)=a \xi^{2}-2 c \xi-b=0$, which are $\xi_{1}>0, \xi_{2}<0$; and we take a contour as shown in figure 2 .

Performing the integration of $\Phi y_{1} d \xi$ we have

$$
\oint y_{1} d \xi=-\frac{1}{2} \oint\left\{\frac{\xi}{\xi^{2}-1}+\frac{a \xi-c}{a f(\xi)}\right\} d \xi=-\pi i
$$

Therefore, in approximation (1), we have the following solution of the equation in $\xi$,

$$
\oint y d \xi=\oint \sqrt{\frac{-a f(\xi)}{\xi^{2}-1}} d \xi-\frac{h}{2 \pi i}(\pi i)=n_{1} h,
$$

or

$$
\oint \sqrt{\frac{-a f(\xi)}{\xi^{2}-1}} d \xi=\frac{h}{2}
$$

for the lowest state.

By a similar treatment of the equation in $\eta$, we can get

$$
\oint \sqrt{\frac{a \eta^{2}-b}{1-\eta^{2}}} d \eta=\frac{h}{2}
$$

But we shall use (6) and (15) at present. In order to evaluate three constants, an extra condition is introduced by taking the minimum energy of the whole system. 
The integral in (15) can be evaluated exactly by standard transformations, but the following method is simpler.

Let

then

$$
\xi=\xi_{1} \cos ^{2} \theta+\sin ^{2} \theta,
$$

then

$$
J_{1}=\oint \sqrt{\frac{-a f(\xi)}{\xi^{2}-1}} d \xi=2 \sqrt{a} \int_{1}^{\xi_{1}} \sqrt{\frac{\left(\xi_{1}-\xi\right)\left(\xi-\xi_{2}\right)}{(\xi-1)(\xi+1)}} d \xi
$$

$=4 \sqrt{a}\left(\xi_{1}-\xi_{2}\right) \sqrt{\alpha \beta} \int_{0}^{\frac{\pi}{2}}\left(1-\alpha \sin ^{2} \theta\right)^{\frac{1}{2}}\left(1-\beta \sin ^{2} \theta\right)^{-\frac{1}{2}} \sin ^{2} \theta d \theta$ where

$$
\alpha=\frac{\xi_{1}-1}{\xi_{1}-\xi_{2}}, \quad \beta=\frac{\xi_{1}-1}{\xi_{1}+1} .
$$

Since $\alpha$ and $\beta$ are small, we can expand $\left(1-\alpha \sin ^{2} \theta\right)^{\frac{1}{2}}\left(1-\beta \sin ^{2} \theta\right)^{-\frac{1}{2}}$ in series and integrate term by term, the series is found to converge pretty rapidly. Hence:

$$
\begin{aligned}
& J_{1}=\oint y_{0} d \xi= h \sqrt{\lambda}\left(\xi_{1}-\xi_{2}\right) \sqrt{\alpha \beta}\left\{\frac{1}{2}-(\alpha-\beta)\left[\frac{3}{16}\right.\right. \\
&+\frac{5}{128}(\alpha+3 \beta)+\frac{35}{2048}\left(\alpha^{2}+2 \alpha \beta+5 \beta^{2}\right)+\frac{63}{32770}\left(5 \alpha^{3}\right. \\
&\left.\left.\left.+9 \alpha^{2} \beta+15 \alpha \beta^{2}+35 \beta^{3}\right)+\ldots\right]\right\}=\frac{h}{2}+\left(n_{1} h\right)
\end{aligned}
$$

We shall determine the lowest state of $\mathrm{H}_{2}^{+}$by (6) and (17). For a given value of $\lambda$, we can evaluate $\mu$ from (6). The corresponding value of $\rho$ can only be obtained by trying different values of $(2 \rho / \lambda)$ in equation (17). In this way, we get the values of $(2 \rho / \lambda)_{0}$ for different $\lambda$ 's, which are listed in the table. Knowing $\lambda$ and $\rho$, we can calculate the total energy $E^{*}$ immediately.

$$
\frac{E^{*}}{E_{H}}=\frac{4 \lambda}{\rho^{2}}-\frac{2}{\rho}
$$

The above calculation, however, gives $\left(E^{*} / E_{H}\right)$ too high, hence approximation (2) is made as follows:

Taking the terms involving $h^{2}$ in (11), and substituting for $y_{0}$ and $y_{1}$, the expressions in (12) and (13), we have:

$$
\begin{aligned}
\mathscr{S}_{2} d \xi= & \oint \frac{1}{2 y_{0}}\left\{-\frac{2 \xi}{\xi^{2}-1} y_{1}-y_{1}^{2}-y_{1}^{\prime}\right\} d \xi \\
= & \oint\left[-\frac{1}{8\{-a f(\xi)\}^{1 / 2}\left(\xi^{2}-1\right)^{3 / 2}}+\frac{4 a c \xi-3 a^{2}+6 a b+5 c^{2}}{8 a\{-a f(\xi)\}^{3 / 2}\left(\xi^{2}-1\right)^{1 / 2}}\right. \\
& \left.-\frac{10\left(a b c+c^{3}\right) \xi+5(b-a)\left(a b+c^{2}\right)}{8 a\{-a f(\xi)\}^{5 / 2}\left(\xi^{2}-1\right)^{1 / 2}}\right] d \xi .
\end{aligned}
$$


Since we know that

$$
\begin{aligned}
\oint \frac{d x}{\left\{-\left(x-x_{1}\right)\left(x-x_{2}\right)\left(x-x_{3}\right)\left(x-x_{4}\right)\right\}^{1 / 2}} \\
=2 \int_{x 2}^{x_{1}} \frac{d x}{\{\}^{1 / 2}}=\frac{4}{\left\{\left(x_{1}-x_{3}\right)\left(x_{2}-x_{4}\right)\right\}^{1 / 2}} K(k),
\end{aligned}
$$

where

$$
x_{1}>x_{2}>x_{3}>x_{4}, k^{2}=\frac{\left(x_{1}-x_{2}\right)\left(x_{3}-x_{4}\right)}{\left(x_{1}-x_{3}\right)\left(x_{2}-x_{4}\right)},
$$

and that the different terms of $y_{2}$ in (19) are the derivatives of $\left\{\left(-\xi^{2}+\alpha \xi+\beta\right)\left(\xi^{2}-\gamma^{2}\right)\right\}^{-1 / 2}$ with respect to $\alpha, \beta$ or $\gamma$, multiplied by constant coefficients, we set up the correspondence $\xi_{1} \sim x_{1}, \gamma \sim x_{2}$, $\xi_{2} \sim x_{3},-\gamma \sim x_{4}$. We then get the different terms in (19) by suitable differentiations of (20). Furthermore, in the result so obtained, we notice that $k^{2}=\left(\xi_{1}-1\right)\left(\xi_{2}+1\right) / 2\left(\xi_{1}-\xi_{2}\right)$. Now this quantity is small, hence we can expand $K(k)$ and $E(k)$ in series and neglect the high powers of $\left(k^{2}\right)$. In this way, we arrive at the following result:

$$
\begin{aligned}
\frac{h^{2}}{4 \pi^{2}} \oint_{y_{2}} d \xi= & \frac{h}{64 \sqrt{2 \lambda} k_{1}^{2}}\left\{\frac{3}{X}+\frac{2}{X^{3}}\right\} \\
& \quad+\frac{h \cdot 3 \rho^{2}}{4 \sqrt{2} \lambda^{5 / 2} k_{1}^{2}} \frac{1}{X^{5}}+\frac{h\left(5 \rho^{2}+6 \lambda \mu-3 \lambda^{2}\right)}{32 \sqrt{2} \lambda^{5 / 2} k_{1}^{2}}\left\{\frac{1}{X^{3}}+\frac{6}{X^{5}}\right\} \\
-\frac{h .5\left(\lambda \mu+\rho^{2}\right) \rho^{2}}{24 \sqrt{2} \lambda^{1 / 2} k_{1}^{2}} & \left\{\frac{15}{8 X^{7}}+\frac{105}{4 X^{9}}\right\} \\
& +\frac{h .5(\lambda-\mu)\left(\lambda \mu+\rho^{2}\right)}{24 \sqrt{2} \lambda^{7 / 2} k_{1}^{2}}\left\{\frac{9}{32 X^{5}}+\frac{15}{8 X^{7}}+\frac{105}{8 X^{9}}\right\}, \quad(21)
\end{aligned}
$$

where

$$
X=\left(\xi_{1}-\xi_{2}\right)^{\frac{1}{2}} \quad, \quad k_{1}^{2}=1-k^{2} .
$$

In approximation (2), the quantum condition now becomes

$$
J_{1}=\oint y_{0} d \xi=\frac{h \sqrt{\lambda}}{2 \pi} \oint \sqrt{\frac{-f(\xi)}{\xi^{2}-1}} d \xi=\frac{h}{2}+\frac{h^{2}}{4 \pi^{2}} \oint y_{2} d \xi+\left(n_{1} h\right) \text {. }
$$

\begin{tabular}{cccccccc}
$\lambda$ & \multicolumn{7}{c}{ TABLE } \\
$\lambda$ & $(2 \rho / \lambda)_{0}$ & $\Delta\left(J_{1} / h\right)$ & $\Delta(2 \rho / \lambda)$ & $\rho$ & $4 \lambda / \rho^{2}$ & $2 / \rho$ & $E^{*} / E_{H}$ \\
1.200 & 1.986 & 0.1190 & 0.302 & 1.372 & 2.550 & 1.459 & 1.091 \\
1.900 & 1.721 & 0.0983 & 0.198 & 1.823 & 2.287 & 1.097 & 1.190 \\
2.200 & 1.646 & 0.0915 & 0.172 & 2.000 & 2.200 & 1.000 & 1.200 \\
2.500 & 1.583 & 0.0871 & 0.154 & 2.171 & 2.121 & 0.921 & 1.200 \\
4.000 & 1.374 & 0.0703 & 0.102 & 2.952 & 1.836 & 0.678 & 1.158
\end{tabular}

If we compare (22) with (17), the only difference is $\Delta J_{1}=\frac{h^{2}}{4 \pi^{2}} \oint y_{2} d \xi$. 
This $\Delta J_{1}$ would introduce an increment $\Delta(2 \rho / \lambda)$ in $2 \rho / \lambda$. If $\Delta J_{1}$ is not too large, we can assume that $\Delta(2 \rho / \lambda)$ is proportional to $\Delta J_{1}$ for each set of values $(\lambda, \mu)$ and so interpolate the value of $2 \rho / \lambda$. Using $\lambda, \mu$ and $\rho_{0}$ in (21), we calculate $\Delta J_{1}$ and then $\Delta(2 \rho / \lambda)$; the result is shown in the table. In the calculation of $\Delta J_{1}$ we only use $\rho_{0}$, this introduces a small error; however, we do not apply further correction.

The above values are plotted, and a solid curve is drawn through them; for comparison, Burrau's result is given in a dotted line (Fig. 3). Our minimum occurs at $\rho=2.08, E^{*} / E_{H}=1.201$ (or 0.04 volts higher than his). The present result therefore agrees with the experimental value within the limits of error; ${ }^{7}$ however, this is perhaps fortuitous.

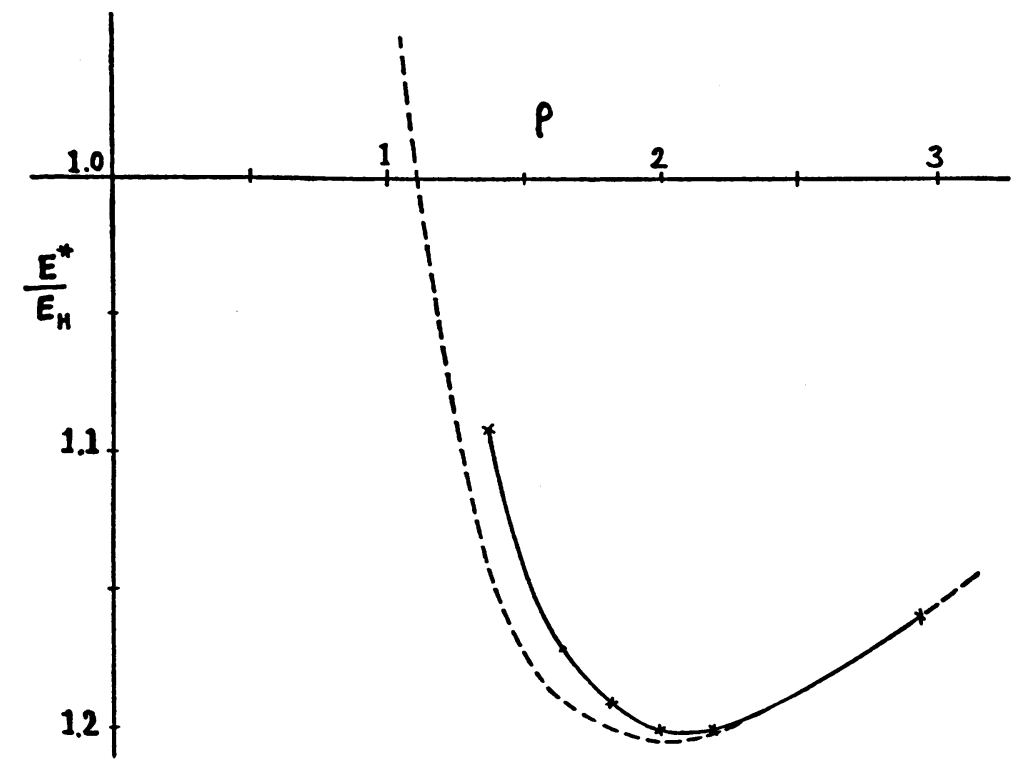

FIGURE 3

In connection with half-quantum numbers, we will also consider the adiabatic transition in the gradual separation of the two nuclei. From (22) and (21) we see that the quantum condition for $\xi$ can be written:

$\lambda^{1 / 2} \oint \sqrt{\frac{-f(\xi)}{\xi^{2}-1}} d \xi+\lambda^{-1 / 2} f_{1}+\lambda^{-3 / 2} f_{2}+\ldots=2 \pi\left(\frac{1}{2}+n_{1}\right)$.

Similarly, we have for $\eta$,

$\lambda^{1 / 2} \oint \sqrt{\frac{\eta^{2}-\mu / \lambda}{1-\eta^{2}}} d \eta+\lambda^{-1 / 2} g_{1}+\lambda^{-z / 2} g_{2}+\ldots=2 \pi\left(\frac{1}{2}+n_{2}\right)$.

When $\rho \longrightarrow \infty, 4 \lambda / \rho^{2}$ must remain finite since it represents the elec- 
tronic part of energy, so $\sqrt{\lambda} \longrightarrow \infty$. In order to have the left side of (24) finite, we must have $\mu / \lambda \longrightarrow 1$. Hence, as $\rho \longrightarrow \infty, \rho / \lambda \longrightarrow 0$ and $\mu / \lambda \rightarrow 1$, and so $f_{1}, f_{2}, \ldots, g_{1} \ldots$ are all bounded, it follows that we have only to consider the first term in (23) and (24).

Since $\mu / \lambda=k_{0}^{2} \longrightarrow 1$, from (24) we have

$$
\begin{aligned}
\sqrt{\lambda} \oint \sqrt{\frac{\eta^{2}-k_{0}^{2}}{1-\eta^{2}}} d \eta & =2\left\{E\left(k_{0}^{\prime}\right)-k_{0}^{2} K\left(k_{0}^{\prime}\right)\right\} \sqrt{\lambda}=\frac{\pi}{2} k_{0}^{\prime 2} \sqrt{\lambda} \\
= & \frac{\pi(\lambda-\mu) \sqrt{\lambda}}{2 \lambda}=\frac{\pi(\lambda-\mu)}{2 \sqrt{\lambda}}=\pi\left(1+2 n_{2}\right) .
\end{aligned}
$$

Since $2 \rho / \lambda \longrightarrow 0, \mu / \lambda \longrightarrow 1$, from (23) we have

$$
\begin{aligned}
\sqrt{\lambda} & \oint \sqrt{\frac{-f(\xi)}{\xi^{2}-1}} d \xi=2 \sqrt{\lambda} \int_{1}^{\xi_{1}} \sqrt{\frac{\left(\xi_{1}-\xi\right)\left(\xi-\xi_{2}\right)}{(\xi-1)(\xi+1)}} d \xi \\
& =2 \sqrt{\lambda} \int_{1}^{\xi_{1}} \sqrt{\frac{\xi_{1}-\xi}{\xi-1}} d \xi=2 \sqrt{\lambda}\left\{-\left(1-\xi_{1}\right) \tan ^{-1} \sqrt{\frac{\xi-1}{\xi_{1}-\xi}}\right\}_{1}^{\xi_{1}} \\
& =\sqrt{\lambda}\left(\xi_{1}-1\right) \pi=\sqrt{\lambda}\left\{\frac{\rho}{\lambda}+\sqrt{\frac{\mu}{\lambda}}-1\right\} \pi=\pi\left(1+2 n_{1}\right) .
\end{aligned}
$$

Let $\lambda-\mu=\lambda \delta$; from (25) and (26) we have

$$
\begin{gathered}
\frac{\lambda-\mu}{\sqrt{\lambda}}=\frac{\lambda \delta}{\sqrt{\lambda}}=\sqrt{\lambda} \delta=2\left(1+2 n_{2}\right), \\
\sqrt{\lambda}\left\{\frac{\rho}{\lambda}+\sqrt{\frac{\mu}{\lambda}}-1\right\}=\sqrt{\lambda}\left\{\frac{\rho}{\lambda}+(1-\delta)^{\frac{1}{2}}-1\right\} \\
=\sqrt{\lambda}\left\{\frac{\rho}{\lambda}-\frac{1}{2} \delta\right\}=\left(1+2 n_{1}\right), \\
\therefore \frac{\rho}{\sqrt{\lambda}}=\frac{1}{2} \sqrt{\lambda} \delta+\left(1+2 n_{1}\right)=\left(1+2 n_{2}\right)+\left(1+2 n_{1}\right)=2\left(1+n_{1}+n_{2}\right), \\
\therefore \frac{E^{*}}{E_{H}} \doteq \frac{4 \lambda}{\rho^{2}}=\frac{1}{\left(1+n_{1}+n_{2}\right)^{2}}
\end{gathered}
$$

Equation (27) gives the adiabatic transition of the energy levels when $\mathrm{H}_{2}^{+}$is dissociated into a nucleus and a $\mathrm{H}$-atom.

In conclusion, the following points are to be mentioned. The restricted old quantum theory does not even give the approximate result; two halfquantum numbers are introduced in the approximation (1) by the polynomial method, i.e., $(1 / 2,1 / 2,0)$ for the lowest state; this gives a fairly good energy value but a nuclear distance which is too big. (Niessen used the quantum numbers $(0,1 / 2,1)$.) The present method gives results very close to those of Burrau for the lowest state; moreover, it can be applied 
to higher states without further complication but the convergence is still doubtful.

I am very much indebted to Professor A. Sommerfeld, Professor P. S. Epstein and Professor $\mathrm{H}$. Bateman for the encouragement and suggestions they have given me in the study of this problem. I wish also to express my gratitude to the China Foundation which provides me with a research fellowship.

${ }^{1}$ Pauli, Ann. Physik, 68, 177, 1922.

2 Niessen, Zeit. Physik, 43, 694, 1927.

3 Burrau, K. Danske Vid. Selskab, V. 7, N. 14, 1927.

4 A. H. Wilson, Proc. Roy. Soc., London, A., 118, 617, 635, 1928.

5 Brillouin, Comptes Rendus, 183, 24, 1926; Wentzel, Zeit. Physik, 38, 518, 1926; Sommerfeld, Wellenmechanischer Ergänzungsband, p. 158, 1929.

- Alexandrow, Ann. Physik, 81, 603, 1926; A. H. Wilson, loc. cit.

7 Birge, Proc. Nat. Acad. Sci. 14, 12, 1928.

\section{NOTE ON THE VELOCITIES AND MAGNITUDES OF EXTERNAL GALAXIES}

\section{By HARLOW SHAPLEY}

\section{Harvard College Observatory, Cambridge, Massachusetts}

Communicated June 13, 1929

The line displacement in the spectra of the extra-galactic nebulae and its relation to distance will continue to be of much significance in the general problems of cosmogony, whether the displacement be interpreted as an indication of radial motion with respect to the observer or mainly as a consequence of fundamental properties of space-time. However optimistic, we must admit much uncertainty in measuring the distances of any but the nearest of the external systems. We have reasons to believe that the relative distances of the various clouds of galaxies may soon be determined with a relatively small percentage error; but the distances to the individual scattered galaxies will lack in certainty because of the wide dispersion in real dimensions, in constitution and structural details, and in total intrinsic luminosity, which make the use of the necessary photometric methods inconclusive.

1. In one of the first general considerations of the velocities of extragalactic nebulae, I pointed out some ten years ago the systematic recession of these objects on both sides of our Galaxy ${ }^{1}$ and the fact that "The speed of spiral nebulae is dependent to some extent upon apparent brightness, indicating the relation of speed to distance or, possibly, to mass."

Six of the brightest spirals had a mean velocity of about fifty kilometers 